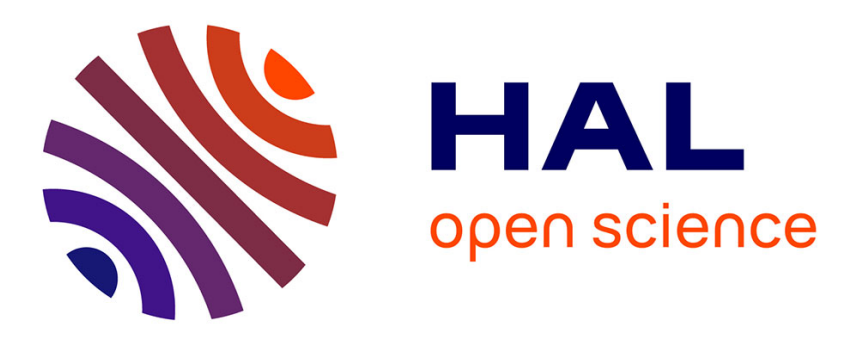

\title{
Diode Schottky sur diamant CVD. Simulation, réalisation technologique et étude de protection périphérique
}

Sojan Koné, Hui Ding, Fabien Thion, Henri Schneider, Karine Isoird, Marie-Laure Locatelli, Dominique Planson, Jocelyn Achard, Riadh Issaoui

\section{To cite this version:}

Sojan Koné, Hui Ding, Fabien Thion, Henri Schneider, Karine Isoird, et al.. Diode Schottky sur diamant CVD. Simulation, réalisation technologique et étude de protection périphérique. European Journal of Electrical Engineering, 2011, 14 (5), pp.553 - 567. 10.3166/ejee.14.553-567 . hal-01627761

\section{HAL Id: hal-01627761 https://hal.science/hal-01627761}

Submitted on 20 Feb 2018

HAL is a multi-disciplinary open access archive for the deposit and dissemination of scientific research documents, whether they are published or not. The documents may come from teaching and research institutions in France or abroad, or from public or private research centers.
L'archive ouverte pluridisciplinaire HAL, est destinée au dépôt et à la diffusion de documents scientifiques de niveau recherche, publiés ou non, émanant des établissements d'enseignement et de recherche français ou étrangers, des laboratoires publics ou privés. 


\title{
Diode Schottky sur diamant CVD - simulation, réalisation technologique et étude de protection périphérique / CVD diamond Schottky barrier diode - simulation, carrying out and edge termination techniques
}

\author{
Sodjan Koné*,** - Hui Ding*,** - Fabien Thion**,*** \\ Henri Schneider*,** - Karine Isoird*** - Marie Laure \\ Locatelli**,**** Dominique Planson*** - Jocelyn Achard***** \\ - Riadh Issaoui $* * * * *$ \\ * CNRS ; LAAS ; 7 avenue du Colonel Roche, F-31077 Toulouse \\ ** Université de Toulouse ; UPS ; INSA ; INP ; ISAE ; LAAS ; F-31077 Toulouse \\ *** Université de Lyon; INSA de Lyon ; Laboratoire AMPERE ; CNRS \\ 21, avenue Jean Cappelle, F-69621 Villeurbanne Cedex \\ **** LAPLACE (Laboratoire Plasma et Conversion d'Energie) \\ 118, route de Narbonne, F-31062 Toulouse Cedex 9 \\ ***** CNRS ; LIMHP ; Université Paris 13 \\ 99, avenue JB Clément, F-93430 Villetaneuse
}

\begin{abstract}
RÉSUMÉ. Le diamant est un semi-conducteur grand gap aux propriétés exceptionnelles qui en font un matériau idéal pour l'électronique de puissance. Son utilisation devrait permettre le développement d'une nouvelle génération de dispositifs avec des performances jamais atteintes à ce jour, notamment dans le domaine des applications à forte puissance etlou haute température. Dans cet article, nous présentons le développement de la filière technologique nécessaire à la réalisation de composants sur diamant. Nous présentons ici l'état actuel de nos travaux selon deux axes; d'une part la plate-forme de simulation développée sur le support Sentaurus TCAD, et d'autre part le développement de différentes briques technologiques nécessaire à la réalisation des premières diodes Schottky. Ces résultats nous permettent de discuter de la qualité du matériau et de nos procédés.

ABSTRACT. Diamond is a wide band gap semiconductor material with outstanding properties for power electronic applications. Its use as an electronic material should allow the rise of a new generation of devices with performance ever achieved to date, particularly in high power and/or high temperature application field. In this paper, we present the development of diamond processing technology for high power electronic devices. The current state of work
\end{abstract}


is illustrated by p-type CVD diamond Schottky barrier diode Sentaurus TCAD simulations, carrying out and an extensive study of edge termination techniques. The material quality and our processes are discussed in light of the obtained results.

MOTS-CLÉS : diamant, grand gap, électronique de puissance.

KEYWORDS: diamond, wide bandgap, power electronic.

DOI:10.3166/EJEE. Volume X . page1-pagefin @ 2011 Lavoisier, Paris

\section{Introduction}

Le diamant est un semi-conducteur grand gap aux propriétés exceptionnelles qui en font un matériau idéal pour l'électronique de puissance. Sa large bande interdite $\left(\mathrm{E}_{\mathrm{s}} \sim 5,5 \mathrm{eV}\right)$ et sa conductivité thermique exceptionnelle $(20 \mathrm{~W} / \mathrm{cm} . \mathrm{K})$ sont bien adaptées aux composants opérant à haute température. En outre, le champ de rupture diélectrique très élevé $(10 \mathrm{MV} / \mathrm{cm})$ et la grande mobilité des porteurs $(2200 \mathrm{~cm} / \mathrm{V} . \mathrm{s}$ pour les électrons (Tranchant, 2007) et $2000 \mathrm{~cm} / \mathrm{V}$.s pour les trous (Teraji, 2004) dans le diamant sont très attractifs pour le concept de composants haute tension et haute fréquence.

Malgré des décennies de recherche, l'utilisation du diamant en électronique demeure très problématique à ce jour. Cela est largement dû à la grande difficulté, d'une part, de synthétiser le diamant monocristallin de qualité électronique, et d'autre part, de trouver des dopants convenables pour le diamant (en particulier donneurs). Le dopage $\mathrm{p}$ au bore est efficace avec une énergie d'activation de $0,37 \mathrm{eV}$ (Volpe et al., 2009). Mais le type n est loin d'être accessible, ce qui limite l'utilisation du diamant en électronique au développement de dispositifs unipolaires.

Dans cet article, nous présentons les travaux que nous menons pour développer une filière technologique diamant. Cela passe par la mise en place d'une plate-forme de simulation sur la base de Sentaurus TCAD et par le développement des différentes briques technologiques nécessaires à la réalisation de dispositifs. Nos travaux sont illustrés par l'étude d'une diode Schottky $1200 \mathrm{~V}$ à forte densité de courant $\left(300 \mathrm{~A} / \mathrm{cm}^{2}\right)$ à haute température $\left(300{ }^{\circ} \mathrm{C}\right)$.

\section{Plate-forme de simulation}

\subsection{Modèles et paramètres}

Nous introduisons les modèles et les paramètres pour la simulation de dispositifs en diamant sur la plate-forme de conception Sentaurus-TCAD. $\mathrm{Du}$ fait que le diamant est un nouveau matériau, nous avons besoin de créer un nouveau fichier de paramètres électriques pour la simulation. Ensuite, chaque modèle y compris l'ensemble de ses paramètres, a besoin d'être spécifié pour simuler avec précision le comportement d'un composant de puissance à base de diamant. L'étude des modèles physiques et les paramètres à $\mathrm{T}=300^{\circ} \mathrm{K}$ a été publiée 
précédemment (Koné et al., 2009). Les principaux modèles comprennent $\mathrm{Nc}, \mathrm{N}_{\mathrm{v}}, \mathrm{n}_{\text {, }}$, $\mathrm{E}_{\mathrm{s}}$ (Ding et al., 2010), le modèle d'ionisation incomplète (Koné et al., 2009) et le modèle de mobilité des trous. Le diamant étant spécialement destiné au fonctionnement à haute température, nous avons travaillé sur un modèle et les paramètres qui permettent de simuler les composants jusqu'à $700^{\circ} \mathrm{K}$.

Modèle de la mobilité en fonction de la concentration et de la température

Ici nous présentons le modèle de mobilité des trous et les paramètres pour une plage de température étendue entre $300^{\circ} \mathrm{K}$ et $700^{\circ} \mathrm{K}$. Nous utilisons le modèle de Bologne développé pour simuler des dispositifs en silicium dans la gamme $\left(300^{\circ} \mathrm{K}-\right.$ $\left.1246^{\circ} \mathrm{K}\right)$. L'intérêt de ce modèle est que la plupart des paramètres dépendent de la température. Le modèle de Bologne est exprimé par les équations [2], [4].

$$
\begin{aligned}
& \mu_{L}(T)=\mu_{\max }\left(\frac{T}{300}\right)^{-\gamma+c\left(\frac{T}{300}\right)} \\
& \mu_{d o p}(T)=\mu_{0}(T)+\frac{\mu_{L}(T)-\mu_{0}(T)}{1+\left(\frac{N_{D, 0}}{C_{r 1}(T)}\right)^{\alpha}+\left(\frac{N_{A, 0}}{C_{r 2}(T)}\right)^{\beta}} \\
& \mu_{0}(T)=\frac{\mu_{0 d} N_{D, 0}+\mu_{0 a} N_{A, 0}}{N_{t o t}}
\end{aligned}
$$




\begin{tabular}{|c|c|c|}
\hline Symbole & Valeur & Unité \\
\hline$\mu_{0 a}$ & $20 \times\left(\frac{\mathrm{T}}{300}\right)^{1.1}$ & $\mathrm{~cm}^{2} / \mathrm{V} . \mathrm{s}$ \\
\hline$C_{r 2}$ & $4.5 \times 10^{17}\left(\frac{T}{300}\right)^{3.5}$ & $\mathrm{~cm}^{2} / \mathrm{V} . \mathrm{s}$ \\
\hline$\mu \max$ & 2000 & $\mathrm{~cm}^{2} / \mathrm{V} . \mathrm{s}$ \\
\hline$\beta$ & 0.96 & 1 \\
\hline$\gamma$ & -1 & 1 \\
\hline $\mathrm{c}$ & -2.6 & 1 \\
\hline
\end{tabular}

Tableau 1. Paramètres du modèle de mobilité pour le diamant dans la gamme $\left(300-700^{\circ} \mathrm{K}\right)$

Une étude bibliographique a été effectuée afin de déterminer les paramètres du modèle de Bologne pour le diamant CVD. Ces paramètres sont répertoriés dans le tableau 1.

La figure 1 illustre la variation de la mobilité de trous en fonction de la concentration de bore pour $\mathrm{T}=300^{\circ} \mathrm{K}$ et $\mathrm{T}=500^{\circ} \mathrm{K}$. La correction et la validation de ces paramètres ont été effectuées avec les données expérimentales entre $300^{\circ} \mathrm{K}$ et $700^{\circ} \mathrm{K}$ (Borst et al., 1996 ; Volpe et al., 2009). Ici, il est important de rappeler que $\mathrm{N}_{A}$ est la concentration chimique de bore dans le diamant, différent de la concentration de trous libres participant effectivement à la conduction électrique. 


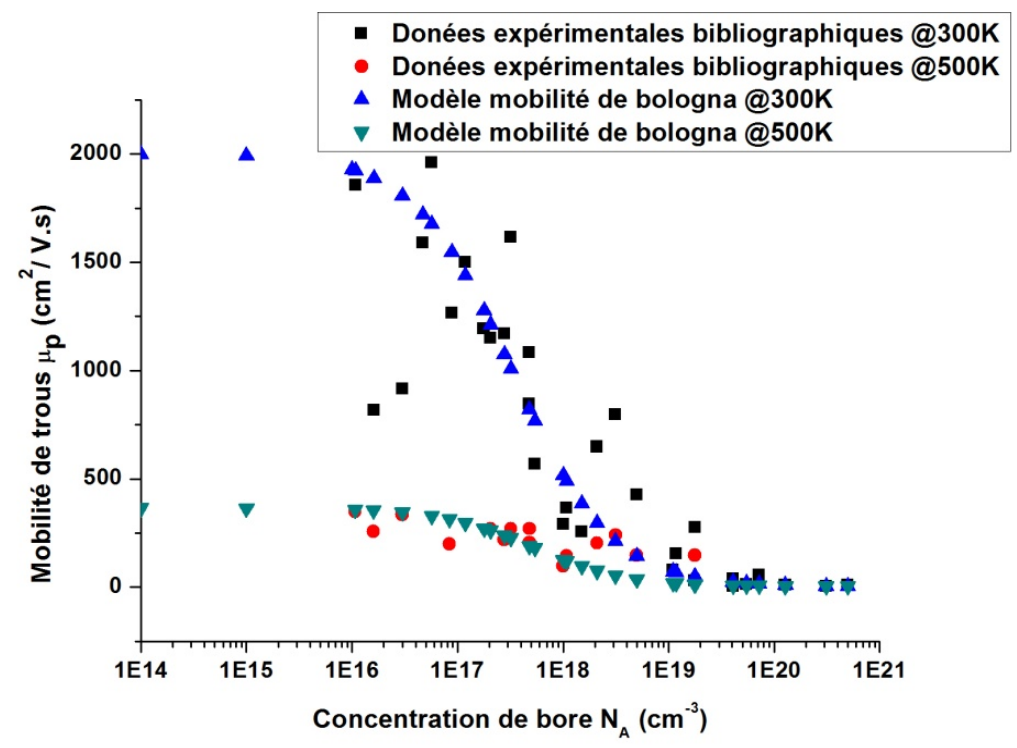

Figure 1. Variation de la mobilité de trous en fonction de la concentration de bore à $T=300^{\circ} \mathrm{K}$ et $\mathrm{T}=500^{\circ} \mathrm{K}$ (modèle de Bologne)

Ces modèles permettent de simuler le comportement en température d'une diode Schottky pseudoverticale telle qu'elle sera réalisée en pratique.

\subsection{Protection périphérique}

Cette même plate-forme de simulation permet d'étudier les protections périphériques nécessaires à la réalisation de composants à haute tenue en tension...

\subsubsection{Principe}

La protection périphérique est une structure implémentée lors du procédé technologique et qui fait partie intégrante de la conception des composants de puissance. Ce dispositif vise à obtenir une tenue en tension proche de celle donnée par la théorie (jonction plane parallèle infinie), en étalant les équipotentielles en périphérie de la structure, ce qui a pour principal effet d'éviter les accroissements locaux du champ électrique dans le composant et qui peut conduire à son claquage prématuré. L'efficacité d'une protection périphérique est évaluée en pourcentage de la tenue en tension théorique, qui est celle de la jonction plane parallèle infinie.

Certaines étapes, telle l'implantation ionique dans le diamant, ne sont pas encore bien maitrisées et empêchent l'utilisation des protections habituelles, tels les anneaux de garde ou encore les JTE (Junction Termination Extension). Il faut donc 
imaginer des protections périphériques basées sur la gravure et des dépôts de couches de diélectriques et de métaux, comme une structure avec plaque de champ (Breazeanu et al., 2006). Les matériaux semi-résistifs présentent aussi des particularités intéressantes et s'imposent comme une alternative intéressante à la minimisation des accroissements locaux du champ électrique au bord du contact Schottky. Il paraît ainsi à propos d'étudier les effets de cette protection et notamment son évolution en température. En effet, on constate que dans le cas de nombreux diélectriques, la résistivité diminue quand la température augmente.

\subsubsection{Plaque de champ sur un diélectrique}

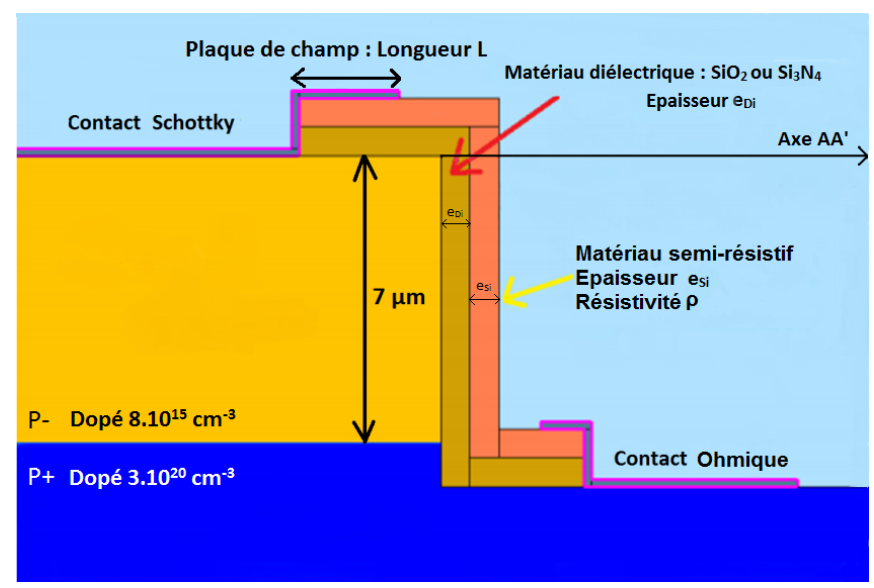

Figure2. Structure pseudo-verticale simulée avec les points d'interrogation représentant les paramètres simulés

La structure envisagée est présentée dans la figure 2. Pour réaliser cette structure quasi verticale, on dépose le contact Schottky sur la couche P- dopée à $8.10^{15} \mathrm{~cm}^{3}$ et épaisse de $7 \mu \mathrm{m}$. Pour réaliser le contact ohmique, on grave l'intégralité de la couche $\mathrm{P}$ afin d'atteindre la couche $\mathrm{P}^{+}$(dopée à $3.10^{\circ} \mathrm{cm}^{s}$ et épaisse de $5 \mu \mathrm{m}$ ). La protection périphérique est réalisée par un dépôt d'un ou plusieurs diélectriques recouvert en partie par le métal de contact. Dans un premier temps, l'étude est réalisée sur un seul diélectrique, puis sur un empilement d'un matériau semi-résistif sur le diélectrique choisi. La tenue en tension idéale (tenue en tension de la jonction plane infinie correspondante) d'une telle diode est de $1500 \mathrm{~V}$ à $600 \mathrm{~K}$. Cette tenue en tension évolue jusque $1990 \mathrm{~V}$ à $800 \mathrm{~K}$, la température à laquelle les simulations sont réalisées afin de faciliter la convergence. En considérant une efficacité de protection périphérique de $80 \%$, on devrait atteindre les $1200 \mathrm{~V}$ désirés pour la structure réelle à $600 \mathrm{~K}$.

La plaque de champ est caractérisée par deux paramètres principaux :

- l'épaisseur du/des diélectrique(s) ; 
- la longueur du recouvrement par le métal du contact sur le diélectrique.

Dans un premier temps, le diélectrique est choisi parmi ceux disponibles dans la salle blanche du LAAS $\left(\mathrm{SiO}_{2}\right.$ et $\left.\mathrm{Si}_{3} \mathrm{~N}_{4}\right)$. Les simulations montrent que plus la permittivité diélectrique du matériau déposé est grande, meilleure est la tenue en tension obtenue. Avec ces paramètres, le résultat des simulations réalisées en utilisant du $\mathrm{SiO}_{2}$ comme diélectrique n'atteint pas les $80 \%$ d'efficacité définis comme objectif. Pour augmenter la tenue en tension, il est nécessaire d'utiliser un diélectrique différent.

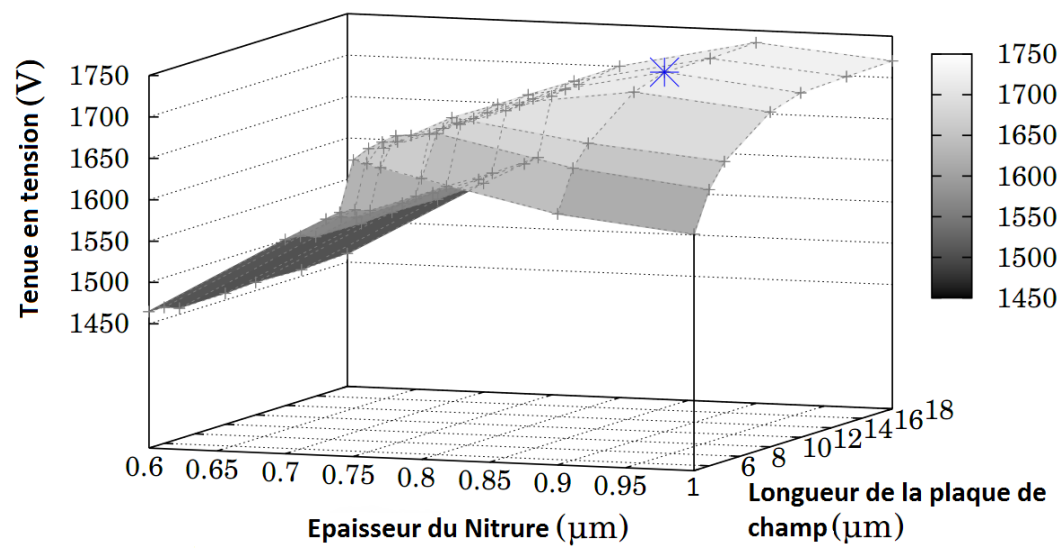

Figure 3. Vue 3D des résultats de simulation de la caractéristique inverse de la diode en diamant protégée par une plaque de champ sur $\mathrm{Si}_{\star} N_{4}$. En (*) la tension maximale obtenue $(1720 \mathrm{~V})$

La figure 4 montre la répartition du champ électrique dans le diamant selon l'axe de coupe (AA'). On remarque deux pics de champ :

- l'un est situé en bord du contact Schottky;

- l'autre est situé à l'extrémité de la plaque de champ.

La configuration est optimale lorsque les deux pics ont la même valeur, ce qui est le cas lorsque la longueur de la plaque est de $10 \mu \mathrm{m}$. Par conséquent, la tenue en tension maximum obtenue est de $1720 \mathrm{~V}$ (soit $85 \%$ d'efficacité, voir figure 3 ) pour une épaisseur de diélectrique de $900 \mathrm{~nm}$ et une plaque de champ de $10 \mu \mathrm{m}$. 
8 European Journal of Electrical Engineering. Volume X- $n^{\circ}$ x/année

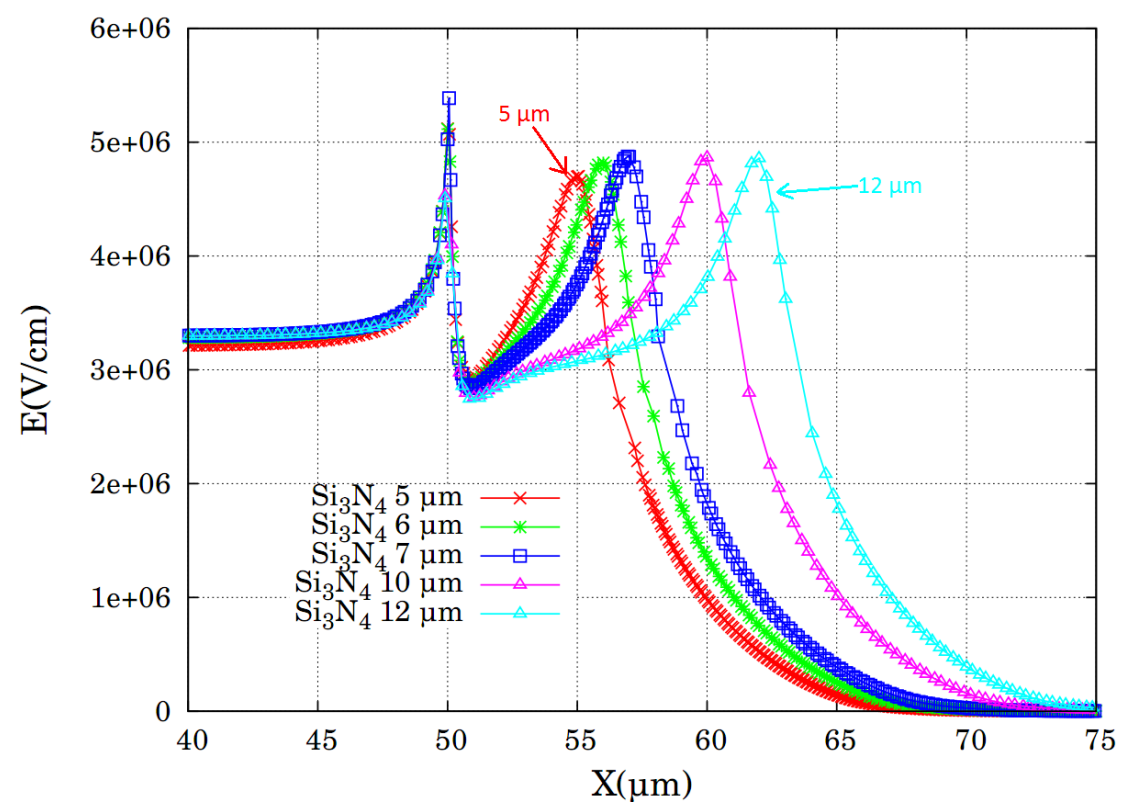

Figure 4. Champ électrique le long de l'axe AA' de la structure précédente. Seule la longueur de plaque de champ varie

Les figures 5 et 6 permettent de constater que l'étalement des équipotentielles n'est pas idéal lors de l'utilisation d'un diélectrique seul. Le maximum du champ électrique est ainsi localisé au bord de la plaque de champ.

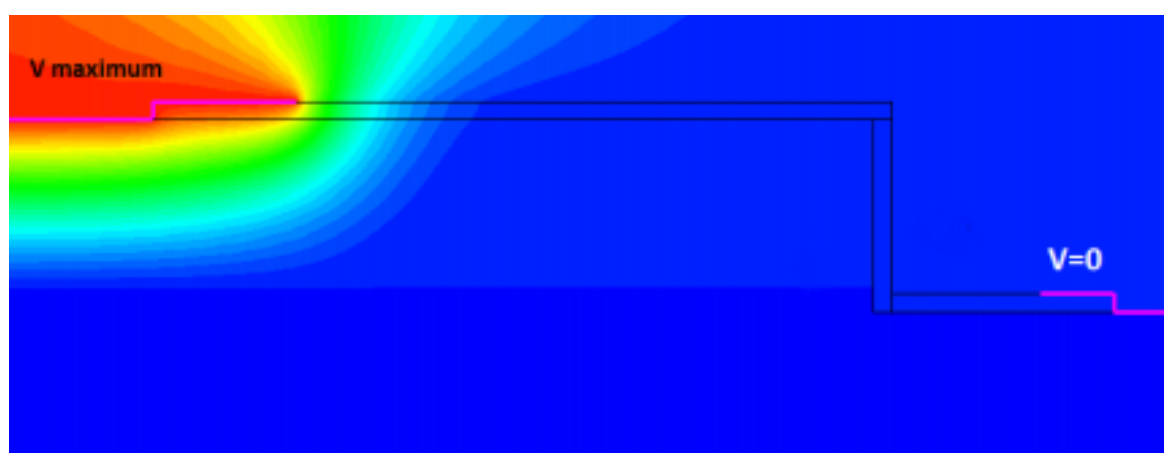

Figure 5. Etalement des équipotentielles dans la structure pour une longueur de plaque de champ de $10 \mu \mathrm{m}$ et une épaisseur de $\mathrm{Si}_{\lessgtr} \mathrm{N}_{+}$de $900 \mathrm{~nm}$ lors du claquage 


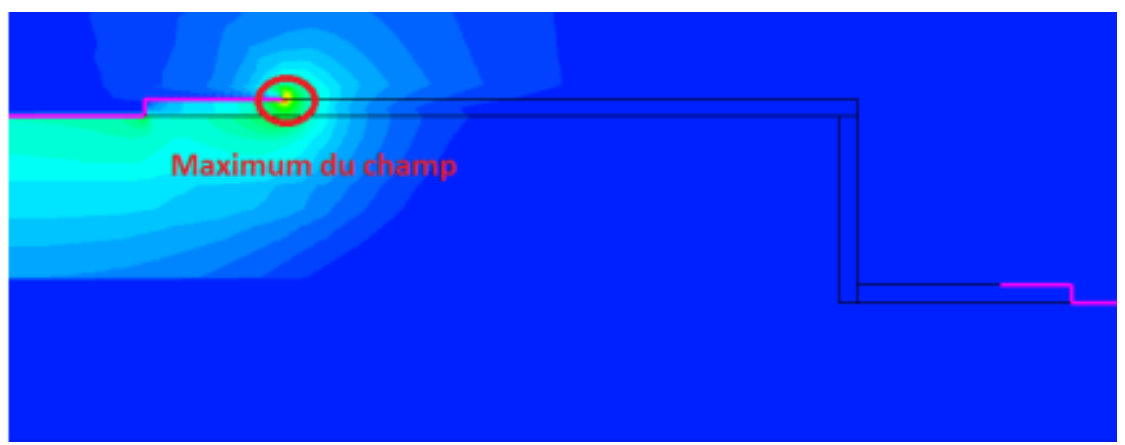

Figure 6. Répartition du champ électrique dans la structure pour une longueur de plaque de champ de 10 um et une épaisseur de $\mathrm{Si}_{\star} N_{4}$ de $900 \mathrm{~nm}$ lors du claquage

\subsubsection{Protection par plaque de champ sur un matériau semi-résistif reposant} sur un diélectrique

La structure simulée est la même que précédemment, avec l'ajout d'une couche d'un matériau semi-résistif sur la couche de $\mathrm{Si}_{3} \mathrm{~N}_{4}$. Les simulations montrent que l'épaisseur du matériau semi-résistif n'influence pas la tenue en tension : seule sa résistivité a un impact sur les niveaux de courants de fuite dans le matériau. La gamme de résistivité typique d'un matériau semi-résistif tel le SiPOS (Silicium polycristallin dopé oxygène) est de $10^{\gamma}-10^{12} \Omega . \mathrm{cm}$, en fonction de la quantité d'oxygène dans le SiPOS et de la température de fonctionnement. Ainsi la tenue en tension est optimisée par la même méthode que précédemment, à savoir en ajustant l'épaisseur de $\mathrm{Si}_{3} \mathrm{~N}_{4}$ et la longueur de la plaque de champ.

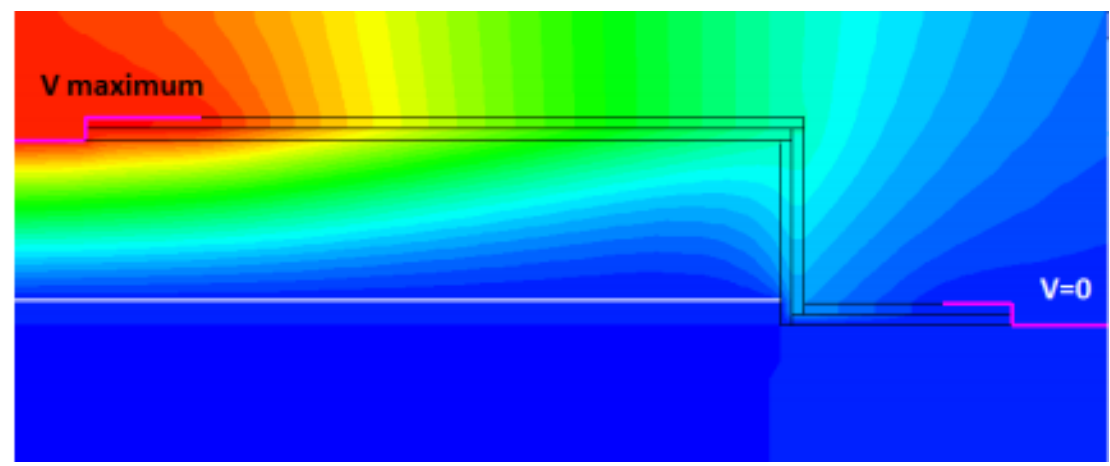

Figure 7. Etalement des équipotentielles dans la structure protégée par l'ajout du matériau semi-résistif pour une longueur de plaque de champ de $10 \mu \mathrm{m}$ et une épaisseur de $\mathrm{Si}_{\mathrm{N}}$ de $500 \mathrm{~nm}$ lors du claquage 
10 European Journal of Electrical Engineering. Volume X- n $\mathrm{x} /$ année

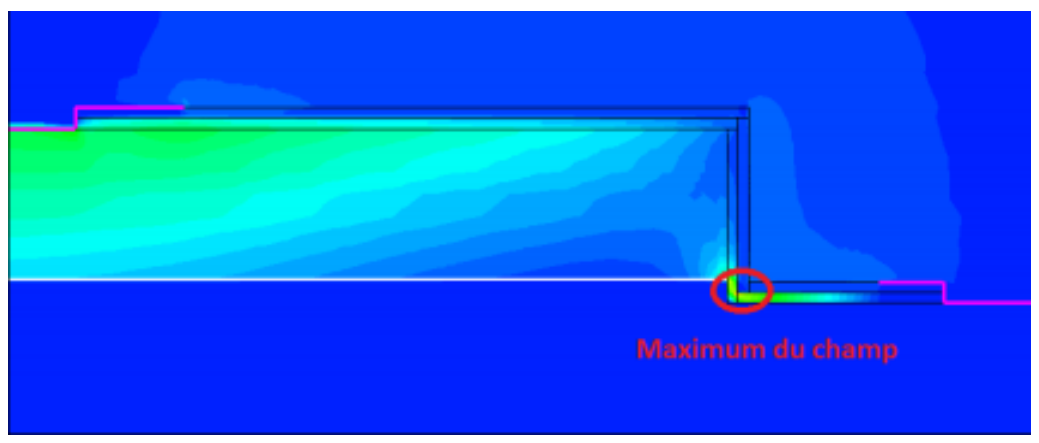

Figure 8. Etalement des équipotentielles dans la structure protégée par l'ajout du matériau semi-résistif pour une longueur de plaque de champ de $10 \mu \mathrm{m}$ et une épaisseur de $\mathrm{Si}_{*}$ de $540 \mathrm{~nm}$ lors du claquage

Les figures 7 et 8 montrent respectivement les équipotentielles et la répartition du champ électrique dans la structure au moment du claquage. On constate un étalement des équipotentielles le long de toute la structure de protection, ce qui contribue à éviter le claquage au bord de la plaque de champ et privilégie celui-ci dans le diamant. Le champ électrique est maximum au pied de la gravure, là où le resserrement des équipotentielles est maximum. Les simulations montrent ainsi que la tension maximum obtenue est de $1840 \mathrm{~V}$, soit une efficacité de $93 \%$, pour une épaisseur de diélectrique de $540 \mathrm{~nm}$ et une plaque de champ ayant une longueur comprise entre 5 et $10 \mu \mathrm{m}$. La longueur de la plaque de champ n'a pas d'impact pour les géométries étudiées, le maximum du champ électrique se produisant au niveau du bord de gravure.

\section{Réalisation technologique}

\subsection{Le dispositif de test}

Sur un substrat diamant synthétique HPHT Ib (100) de $3 \mathrm{~mm}$ x $3 \mathrm{~mm}$, deux couches de diamant de type P ont été déposées par épitaxie CVD. Avant le dépôt, le substrat a subi, en plus du nettoyage chimique classique permettant d'éliminer toutes traces de contaminants métalliques et organiques, un prétraitement par plasma $\mathrm{H}_{2} / \mathrm{O}_{2}$ dont les bénéfices ont déjà largement été démontrés, particulièrement dans le cas de la croissance de films épais (Tallaire et al., 2004).

Une première couche de $6 \mu \mathrm{m}$ d'épaisseur fortement dopée a d'abord été épitaxiée en utilisant une faible densité de puissance micro-onde $\left(50 \mathrm{~W} . \mathrm{cm}^{3}\right.$, $2000 \mathrm{ppm}$ de $\left.(\mathrm{B} / \mathrm{C})_{\mathrm{su}}\right)$ de manière à atteindre la transition métallique. Une deuxième couche de faible dopage a ensuite été déposée à forte densité de puissance $\left(100 \mathrm{~W} . \mathrm{cm}^{3}\right)$ avec un rapport $(\mathrm{B} / \mathrm{C})_{\text {an }}$ de quelques ppm de manière à limiter autant 
que possible le dopage. Les concentrations de bore des zones $\mathrm{P}+(6 \mu \mathrm{m})$ et $\mathrm{P}$ $(1,6 \mu \mathrm{m})$ sont de l'ordre de $4.10^{\mathrm{w}} \mathrm{cm}^{3}$ et $3 \cdot 10^{\mathrm{n}} \mathrm{cm}^{3}$ respectivement.

Afin de réaliser le contact ohmique par dépôt de Ti/Pt/Au sur la couche $\mathrm{P}$, on grave par RIE/ICP la couche P en bordure de l'échantillon. Une distance de $50 \mu \mathrm{m}$ est gardée entre le contact ohmique et les flans de gravure mesa. Le contact obtenu est alors recuit sous atmosphère contrôlée (Azote) à $500^{\circ} \mathrm{C}$ pendant $1 \mathrm{~h}$. Des contacts Schottky circulaires en chrome $(\mathrm{Cr})$ sont ensuite déposés par évaporation thermique sur la zone $\mathrm{P}$ centrale $(2 \mathrm{~mm} \times 2 \mathrm{~mm})$ après traitement de surface à l'oxygène $\left(\mathrm{O}_{2}\right)$. Les longueurs de contact $(\mathrm{L}$ ) utilisées sont $50 \mu \mathrm{m}, 100 \mu \mathrm{m}, 150 \mu \mathrm{m}$ et $200 \mu \mathrm{m}$ (figure 9).
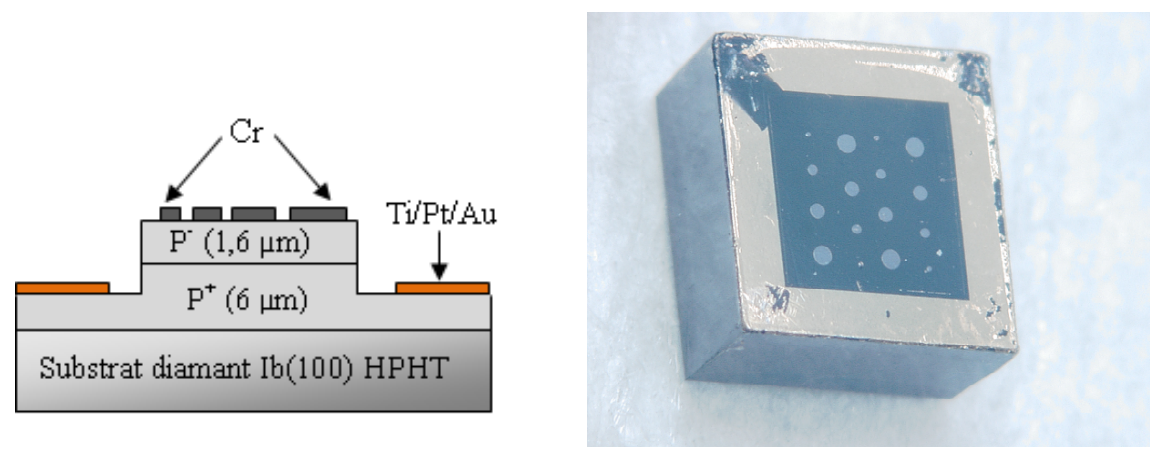

Figure 9. Vue en coupe et photographie 3D de la structure étudiée

\subsection{Caractérisation}

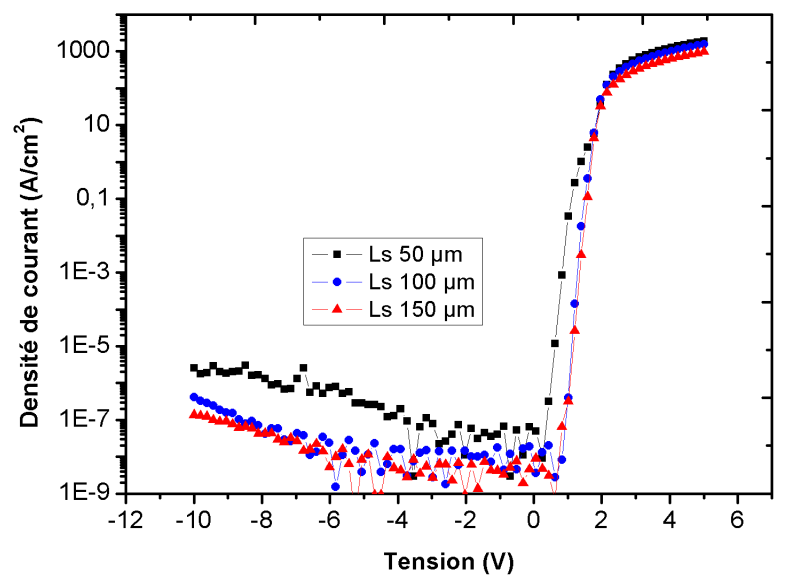

Figure 10. Caractéristiques $I(V)$ des diodes pour chaque longueur de contact 
Les mesures $\mathrm{I}(\mathrm{V})$ ont été effectuées à l'aide d'un analyseur HP $4142 \mathrm{~B}$ à température ambiante $(300 \mathrm{~K})$. Nous présentons les caractéristiques $\mathrm{I}(\mathrm{V})$ des diodes ayant donné le meilleur résultat pour chaque longueur de contact ( $\mathrm{L}_{\text {s }}$ étudiée.

Des densités de courant maximales $2000 \mathrm{~A} / \mathrm{cm}^{2}, 1600 \mathrm{~A} / \mathrm{cm}^{2}$ et $1000 \mathrm{~A} / \mathrm{cm}^{2}$ sont mises en évidence à $5 \mathrm{~V}$ respectivement pour les longueurs de contact de 50 , 100 et $150 \mu \mathrm{m}$ sur la figure 10 . Mais les dispositifs sont très limités sous polarisation inverse. Les mesures de tension de claquage ont été réalisées dans le fluor inerte FC40 et dans l'air (figure 11), ce qui montre que le claquage ne dépend pas de l'environnement, mais apparaît dans le diamant à une tension proche de $55 \mathrm{~V}$. Si l'on considère une répartition triangulaire du champ électrique dans la couche active uniformément dopée, la tension de claquage $\left(\mathrm{V}_{\mathrm{в}}\right)$ et le champ électrique correspondant $\left(\mathrm{E}_{\max }\right)$ pour un composant de puissance unipolaire peuvent être reliés par l'expression (Tolbert et al., 2003) :

$$
V_{B R} \approx \frac{\varepsilon_{s} \cdot E^{2}{ }_{M A X}}{2 q N_{A}}
$$

où $\mathcal{E}$ est la constante diélectrique $\left(F / \mathrm{cm}^{2}\right), E_{\text {wax }}$ le champ électrique maximum au moment du claquage $(V / \mathrm{cm}), q$ la charge élémentaire $(C), N$ le dopage de la couche active (couche P). A partir de [1], le champ électrique lors du claquage est de 1,3 MV/cm pour nos dispositifs. Si l'on se réfère au champ critique théorique du diamant $(10 \mathrm{MV} / \mathrm{cm})$, il est clair que les performances de nos dispositifs en inverse sont bien en dessous de la potentialité attendue du diamant dans les applications haute tension. L'écart important entre les performances expérimentales et théoriques communément rencontré dans la littérature illustre bien que la synthèse du diamant de qualité électronique reste toujours un énorme verrou à lever. Le champ électrique maximum relevé dans la littérature, à ce jour, n'excède pas $3 \mathrm{MV} / \mathrm{cm}$ (Vescan et al., 1998 ; Twitchen et al., 2004). Ces résultats sont néanmoins encourageants compte tenu de la faible maturité du diamant comparée à d'autres matériaux tels que le $\mathrm{SiC}$. 


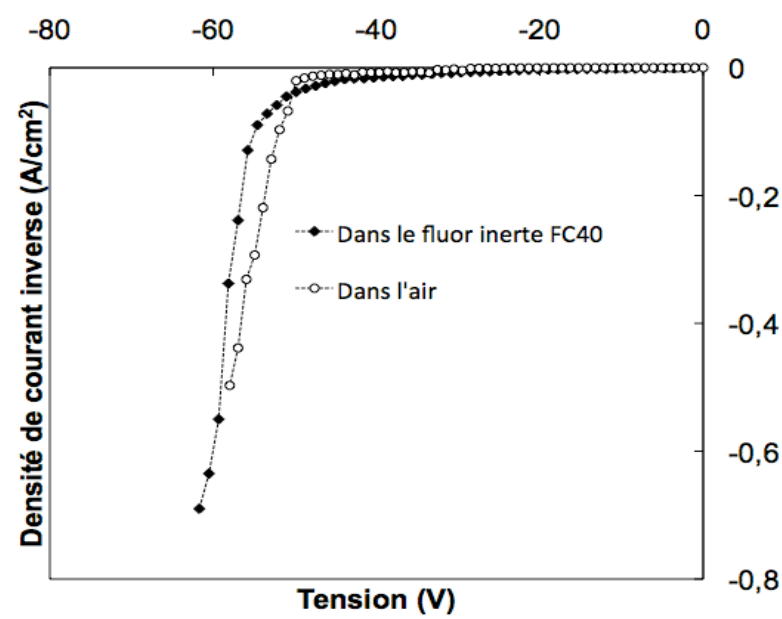

Figure 11. Caractéristique I $(V)$ inverse de la diode Schottky Cr/diamant dans l'air et dans le fluor inerte FC40

La figure 12 montre les différentes diodes réalisées sur un même échantillon, 8 diodes sur 16 fonctionnent correctement. Les diodes les plus larges sont courtcircuitées par des défauts traversant, ce qui conforte l'hypothèse d'une forte densité de défauts.

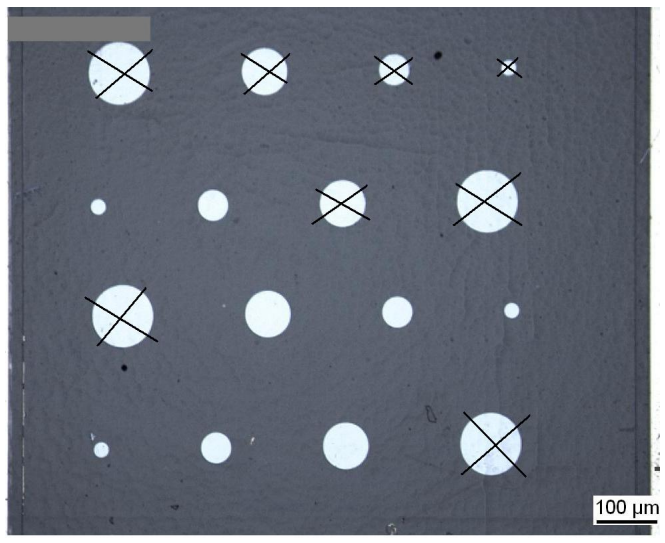

Figure 12. Les dispositifs Schottky Cr/diamant défaillants

\subsection{Comparaison simulation et expérience}

Les simulations ont été effectuées en considérant une compensation nulle $\left(\mathrm{N}_{\mathrm{o}}=0\right)$. 
La figure 13 compare la caractéristique $\mathrm{I}(\mathrm{V})$ expérimentale et la simulation d'une diode de $100 \mu \mathrm{m}$ de diamètre à $\mathrm{T}=300^{\circ} \mathrm{K}$.

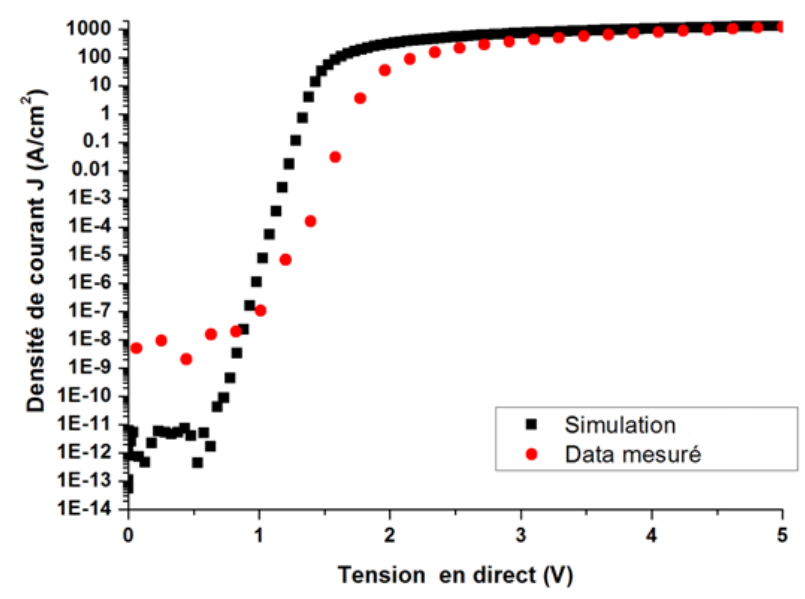

Figure 13. Caractéristiques $I(V)$ directes expérimentale à $300^{\circ} \mathrm{K}$

La densité de courant direct simulée est en bon accord avec la mesure expérimentale. Par contre, le niveau des courants de fuite mesurés avant le seuil est plus élevé. On constate également un écart entre la tension de seuil mesurée et simulée. Les simulations ne prennent pas en compte les effets d'interfaces (Charges, recombinaisons, défauts...) qui peuvent induire des courant de fuite élevés et une variation de la hauteur de barrière Schottky. Dans un contact Schottky réel, une analyse rigoureuse du mécanisme de transport de charges doit tenir compte de l'émission tunnel, à basse température, et des courants de recombinaison dans la zone de charge d'espace dans l'éventualité d'existence de centres de recombinaison dans la bande interdite du semi-conducteur. Ces phénomènes parasites associés aux défauts de volume (dislocations) induisent une augmentation rapide des courants de fuite avec la polarisation inverse conduisant à un claquage prématuré du dispositif. La figure 14 présente les caractéristiques inverses expérimentale et simulée. La concordance ente la limite de claquage expérimentale et simulée monte la pertinence de modèles et paramètres utilisés pour la simulation des dispositifs.

Des travaux sont en cours sur la simulation pour la prise en compte des phénomènes d'interfaces évoqués précédemment et la validation des modèles et paramètres en température. 


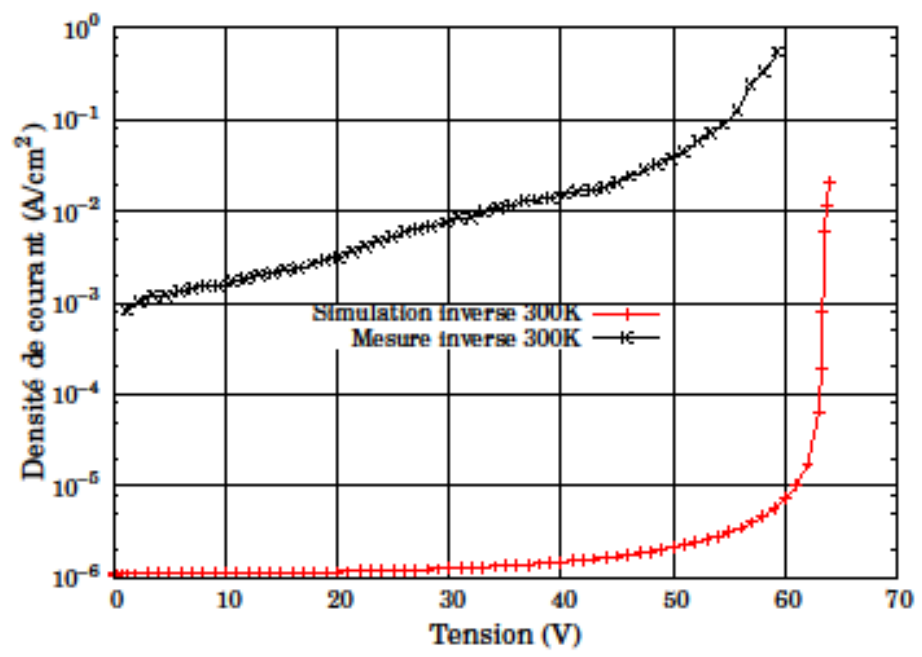

Figure 14. Caractéristiques $I(V)$ inverses expérimentales à $T=300^{\circ} \mathrm{K}$

\section{Conclusion}

Le diamant est un matériau potentiel pour la réalisation de dispositifs électroniques adaptés aux applications forte puissance et haute température. Dans cet article, nous avons présenté des diodes Schottky pouvant fonctionner à forte densité de courant $\left(2000 \mathrm{~A} / \mathrm{cm}^{2}\right)$. Ces résultats montrent la grande potentialité du diamant pour les composants de puissance. Par contre, les performances passables des dispositifs en inverse mettent en évidence l'énorme effort à faire sur la croissance des couches, en termes de diminution de quantité de défauts. La bonne concordance entre simulation et expérience tend à montrer la pertinence des modèles et paramètres employés pour la simulation de dispositif en diamant. Mais il reste toutefois à prendre en compte d'autres phénomènes physiques notamment de surface en choisissant les modèles et paramètres adaptés. Nous travaillons aussi en parallèle sur l'expérience et la simulation afin de valider les modèles et paramètres en température. La protection périphérique basée sur une combinaison de matériau semirésistif reposant sur du nitrure semble prometteuse. Les efficacités obtenues sont respectivement de $85 \%$ pour une plaque de champ sur $\mathrm{Si}_{i} \mathrm{~N}_{\star}$ et de $93 \%$ à l'aide d'un matériau semi-résistif. Les efforts doivent être déployés pour optimiser de manière plus poussée ces structures. Une attention particulière va être apportée aux couches de matériau semi-résistifs et à la gravure des composants. Les études de caractérisation des différents dépôts sont en cours au laboratoire, ainsi qu'une étude plus poussée sur la gravure RIE-ICP du diamant. Des composants nouveaux seront réalisés en tenant compte de toutes les optimisations possibles et protégés de cette manière. 
16 European Journal of Electrical Engineering. Volume X- ${ }^{\circ} \mathrm{x} /$ année

\section{Remerciements}

Ces travaux ont été menés à bien avec le soutien technique de la plate-forme technologique RTB du LAAS/CNRS et du personnel TEAM associé ainsi que le soutient financier du pôle de Compétitivité aéronautique et Espace.

\section{Bibliographie}

Borst T. H., Weis O., "Boron-doped homoepitaxial diamond layers: Fabrication, characterization, and electronic applications", Phys. Status Solidi, vol. 154, n 1, 1996, p. 423-444.

Brezeanu M., Avram M., Rashid S., Amaratunga G.A.J., Butler J.E., Rupesinghe N., Udrea F., Tajani A., Dixon M., Twitchen D., Garraway A., Chamund D., Taylor M.J., Brezeanu G., "Termination structures for diamond schottky barrier diodes", Proceedings of the 18" International Symposium onpower semiconductor devices and IC's, 2006.

Ding H., Isoird K., Schneider H., Kone S., Civrac G., "Basic parameters and models in simulation of CVD diamond devices" Diamond and Related Materials, vol. 19, $\mathrm{n}^{\circ}$ 5-6, 2010, p. 500-502.

Koné S., Ding H., Schneider H., Isoird K., Civrac G., "High performances CVD diamond Schottky barrier diode", EPE 2009, Barcelona Spain, 2009.

Tallaire A., Achard J., Silva F., Sussmann R. S., Gicquel A., Rzepka E., Physica Status Solidi, (a), vol. 201, n 2419, 2004.

Teraji T., Arima K., Wada H., Toshimichi Ito, "High-quality boron-doped homoepitaxial diamond grown by high-power microwave-plasma chemical vapor deposition", Applied Physics Letters, vol. 96, $\mathrm{n}^{\circ}$ 1, 2004, p. 5906-5908.

Tolbert L.M, Ozpineci B, Islam SK, Chinthavali MS., "Wide bandgap semiconductors for utility applications”, Power and Energy Systems, Proceedings, 2003, p. 317-321.

Tranchant N., Nesladek M., Thomson D., Remes Z., Bogdan A., Bergonzo P., “Time of flight study of high performance CVD diamond detector devices", Phys. Stat. Solid., vol. 204, $n^{\circ} 9,2007$, p. 2827-3200.

Twitchen D.J., Whitehead A.J., Coe S.E., Isberg J., Hammersberg J., Wikström T., Johansson E., "High-voltage single-crystal diamond diodes", IEEE Transaction on Electronic Devices, vol. 51, n 5, May 2004, p. 826-828.

Vescan A., Dauville I., Gluche P., Ebert W., Kohn E., "High temperature, High voltage operation of diamond Schottky diode", Diamond and Related Materials, vol. 7, 1998, p. 581-584.

Volpe P.N., Pernot J., Muret P., Omnes F., "High hole mobility in boron doped diamond for power device applications" Applied Physics Letters, vol. 94, n 9, 2009, p. 092102-3. 